\title{
NOVEL ORGANIC SEMICONDUCTOR BTQBT WITH HIGH CONDUCTIVITY AND HIGH MOBILITY
}

\author{
H. INOKUCHI AND K. IMAEDA \\ Institute for Molecular Science, Myodaiji, Okazaki 444, Japan
}

(Received July 18, 1995)

Dedicated to Professors Krzysztof Pigon, Józef W. Rohleder
and Zdzistaw Ruziewicz on the occasion of their 70th birthdays*

We have found a novel organic semiconductor, BTQBT, with a high electrical conductivity of about $10^{-3} \mathrm{~S} \mathrm{~cm}^{-1}$ and a high Hall mobility of about $3 \mathrm{~cm}^{2} \mathrm{~V}^{-1} \mathrm{~s}^{-1}$ at room temperature. We have also succeeded in measuring the temperature dependence of the Hall mobility. It varies with temperature as $T^{-1.6}$, which agrees with the theoretical $T^{-1.5}$ dependence for the mobility determined by lattice scattering. The characteristic transport property of BTQBT results from strong intermolecular interactions in the crystal, which is convinced by the energy dispersion with a bandwidth of about $0.5 \mathrm{eV}$ from the theoretical and experimental band structures.

PACS numbers: $72.20 .-\mathrm{i}, 79.60 .-\mathrm{i}$

\section{Introduction}

Almost all saturated organic compounds are electrical insulators. However, in the 1940's, when polycyclic aromatic compounds, phthalocyanines and dyestuffs, such as cyanine dyes, were major objects of study, the investigation of electrical conductivity of organic compounds was started and "organic semiconductors" was established, as a new field of research.

In the field of organic semiconductors, two major categories of organic solids are known to offer the prospect of electrical conduction. The first group consists of charge-transfer complexes such as the perylene-bromine complex. The value of conductivity of these complexes were quite high among organic materials. Consequently, conductivity measurements on a large number of the charge-transfer

*Submitted on invitation of the Institute of Physical and Theoretical Chemistry, Technical University of Wrocław, Wrocław, Poland. 
complexes (donor-acceptor complexes) have been carried out. The second group comprises single-component materials: typical examples are polycyclic aromatic compounds and also phthalocyanines. Their conductivities, generally speaking, are not so good as those of the first group.

From the view point of the analysis of their conduction mechanism, however, the study of conduction in a single-component system is desirable. Recently, several single-component organic semiconductors liaving fairly good conduction were discovered.

In this report, we will present the study on a single-component organic semiconductor BTQBT (bis $[1,2,5]$ thiadiazolo-p-quinobis(1,3-dithiole)) with fairly high conductivitỷ.

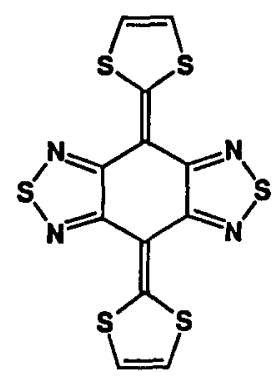

\section{Physical properties of BTQBT}

\subsection{Preparalion}

To increase the conductivity of single-component molecular material, we must prepare molecules with strong intermolecular interactions between adjacent molecules. Following this consideration, we have designed a novel compound, bis[1,2,5] thiadiazolo- $p$-quinobis(1,3-dithiole), in which the electron-withdrawing heterocycles are fused to the skeleton of a very strong electron donor, $2,2^{\prime}$-p-quinobis(1,3-dithiole).

BTQBT was obtained in $48 \%$ yield by a Wittig-Horner reaction of a 2 -methylphosphono-1,3-dithiole [1] with 4II,8II-benzo[1,2-c;4,5- $\left.\mathrm{c}^{\prime}\right]$ bis[1,2,5] thiadiazole$-4,8$-dione [2]. BTQBT has a high melting point $\left(>450^{\circ} \mathrm{C}\right)$ and is sublimed at $350^{\circ} \mathrm{C} / 0.1$ torr. This character shows a strong interaction among molecules in the crystal. The colour of the solid material is reddish-violet and the absorption maxima are found at 470 and $542 \mathrm{~nm}$ in $\mathrm{KBr}$ disk. The solubilities in almost all solvents are very low, while it is a little dissolved in nitrobenzene upon heating.

\subsection{Cryslal structure}

Single crystals of BTQBT have been grown by recrystallization from hot nitrobenzene. The BTQBT crystal belongs to the monoclinic system with the space group $C 2 / m$ and the lattice parameters; $a=16.226(4), b=11.191(2)$, $c=3.833(1) \AA, \beta=96.88(2)^{\circ}, V=691.0(3) \AA^{3}, Z=2, \rho_{\text {calcd }}=1.91 \mathrm{~g} \mathrm{~cm}^{-3}[3]$. 


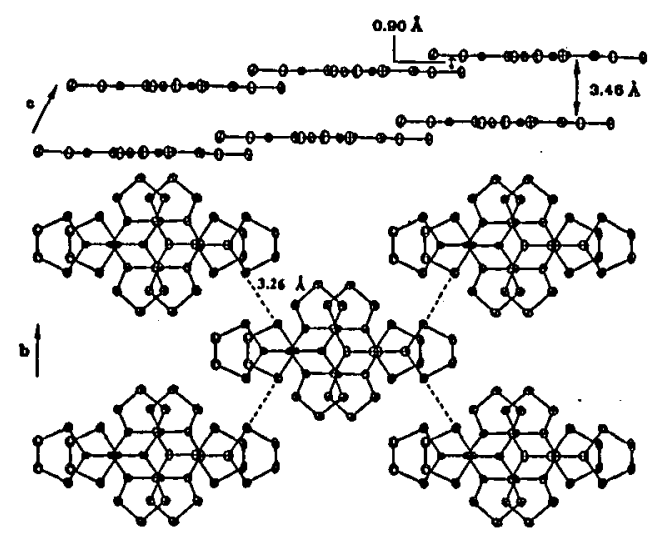

Fig. 1. Crystal structure of BTQBT.

As shown in Fig. 1, the BTQBT molecule is completely planar, suggesting the delocalization of $\pi$-electrons. This planarity results from the intramolecular contact of $2.78 \AA$, which is significantly shorter than the van der Waals ( $v d W)$ S-N distance of $3.55 \AA$, between $S$ atom of the dithiole ring and $N$ atom of the thiadiazole ring. The BTQBT molecules are uniformly stacked along the $c$ axis with an interplanar spacing of $3.46 \AA$ and the effective intermolecular overlaps. In addition, they are arranged with a sheet-like network through a strong S-S contact of $3.26 \AA$ between neighbouring dithiole rings. This value is surprisingly shorter than the vdW S-S distance of $3.70 \AA$. These intermolecular interactions represent the two-dimensional nature of the BTQBT solid.

\subsection{Transport properly}

The BTQBT crystal shows extremely low electrical resistivity $\rho_{c}$ of $1.3 \times$ $10^{3} \Omega \mathrm{cm}$ at room temperature for single-component organic crystals [4]. As shown in Fig. 2, it behaves as a simple semiconductor with $E_{\text {a }}$ (activation energy) = $0.21 \mathrm{eV}$ in the temperature range 110 to $350 \mathrm{~K}$. The anisotropy of the resistivity is found to be $\rho_{c}: \rho_{b}: \rho_{a}=1: 2: 100$. The small ratio of $\rho_{b} / \rho_{c}=2$ is consistent with the two-dimensional intermolecular interaction in the crystal. We have examined the pressure effect on the resistivity. The resistivity at $10 \mathrm{kbar}$ decreases to be only $1 / 5$ of that at 1 bar. The manner of the small change in resistivity by pressure is similar to that in highly conductive charge-transfer complexes and organic metals. This can be qualitatively understood by a small increase in the intermolecular overlap which is initially large in highly conductive organic materials. Such a feeble pressure effect on the resistivity in the BTQBT crystal supports the existence of strong intermolecular interactions through large $\pi$-orbital overlaps between and within the columns.

It is noteworthy that a IIall effect has been observed in the BTQBT crystal $[4,5]$. The detection of a IIall voltage in organic molecular crystals is extremely 


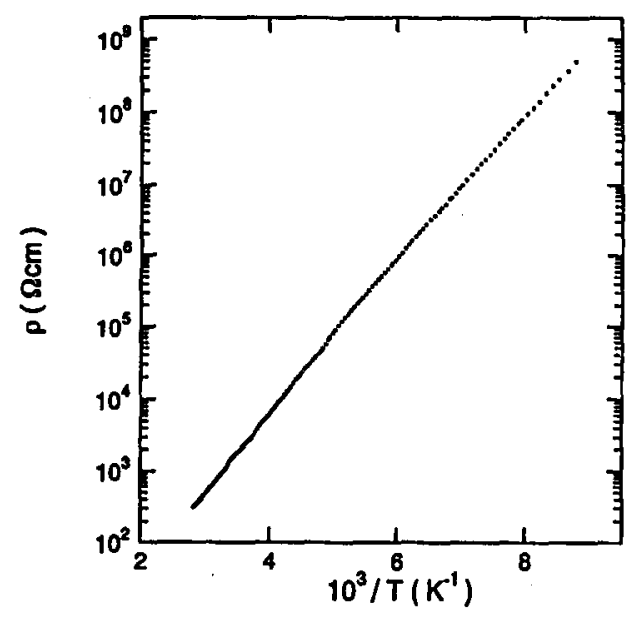

Fig. 2. Temperature dependence of the electrical resistivity of BTQBT crystal.

difficult because of their very high resistivity and small carrier mobility. The observation in the BTQBT crystal is very rare in organic semiconductors. The Hall mobility $\left(\mu_{\mathrm{H}}\right)$ can be expressed by

and

$$
\mu_{\mathrm{H}}=R_{\mathrm{H}} / \rho
$$

$$
R_{\mathrm{H}}=\left(V_{\mathrm{H}} d\right) /\left(I_{x} H_{z}\right)
$$

where $R_{\mathrm{H}}$ is the Hall coefficient, $\rho$ the electrical resistivity, $V_{\mathrm{H}}$ the Hall voltage, $d$ the thickness of the crystal, $I_{x}$ the electric current and $H_{z}$ the magnetic field. For a crystal of $1.7 \mathrm{~mm}$ (length) $\times 0.09 \mathrm{~mm}$ (width) $\times 0.075 \mathrm{~mm}$ (thickness), $V_{\mathrm{H}}$ of $0.46 \mathrm{mV}$ is observed under a constant dc voltage of $30 \mathrm{~V}$ and a magnetic field of $1 \mathrm{~T}$ at room temperature. Using Eqs. (1) and (2), $\mu_{\mathrm{H}}$ is calculated to be about $3 \mathrm{~cm}^{2} \mathrm{~V}^{-1} \mathrm{~s}^{-1}$. This value is considered to be meaningfully large as compared to that smaller than $1 \mathrm{~cm}^{2} \mathrm{~V}^{-1} \mathrm{~s}^{-1}$ in a drift mobility determined by means of the time-of-flight technique for other organic solids $[6,7]$.

We have performed the extended Hückel molecular orbital calculation for the BTQBT crystal [8]. Table summarizes the calculated intermolecular transfer integral $(t)$ between the highest occupied molecular orbitals (HOMOs) and the lowest unoccupied molecular orbitals (LUMOs). The transfer integral between HOMOs is largest $(t=0.123 \mathrm{eV})$ along the stacking direction parallel to the $c$ axis. Figure 3 shows the calculated energy-band structure of the HOMO and LUMO bands. The energy dispersion can be seen clearly along the direction of $B$ to $X$ point, $\Gamma$ to $Z$ point and $A$ to $Y$ point which correspond to the stacking direction. The bandwidth $(W)$ given by $4 t$ in the tight-binding model is estimated to be about $0.5 \mathrm{eV}$. This value is extraordinarily large for molecular organic materials. We have succeeded in direct observation of the energy-band dispersion in a BTQBT oriented thin film by angle-resolved ultraviolet photoelectron spectroscopy (see Chap. 3) [9]. The experimental bandwidth of the HOMO band is found to be about $0.4 \mathrm{eV}$, which is in good agreement with the theoretical one. 
TABLE

Calculated transfer integrals of HOMOs and LUMOs for BTQBT crystal.

\begin{tabular}{c|c|c}
\hline \hline Crystal direction & $\begin{array}{c}\text { IIOMO-IIOMO } \\
{[\mathrm{eV}]}\end{array}$ & $\begin{array}{c}\text { LUMO-LUMO } \\
{[\mathrm{eV}]}\end{array}$ \\
\hline$c$ & 0.123 & 0.100 \\
$a / 2+b / 2$ & 0.021 & -0.004 \\
$a / 2+b / 2-c$ & -0.003 & 0.000 \\
$b$ & -0.016 & 0.023
\end{tabular}

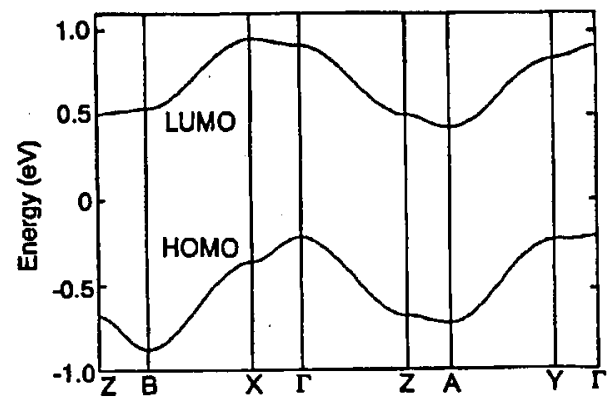

Fig. 3. Calculated energy-band structure of BTQBT crystal.

According to the wide-band model $\left(W>k_{\mathrm{B}} T, k_{\mathrm{B}}\right.$ being the Boltzmann constant), we roughly estimate the mobility of the BTQBT crystal [10]. The carrier mobility is expressed by $\mu=e \tau / m^{*}$, where $\tau$ is the relaxation time and $m^{*}$ the effective mass. With the limiting condition for $\tau>\hbar / W$ given by the uncertainty principle, we obtain the following equation at $290 \mathrm{~K}$ :

$$
\mu>\left(e / m^{*}\right)\left(h / k_{\mathrm{B}} T\right)=20\left(m / m^{*}\right)\left[\mathrm{cm}^{2} \mathrm{~V}^{-1} \mathrm{~s}^{-1}\right] .
$$

The transfer integral $t$ is related to the effective mass $m^{*}$ by $m^{*}=\hbar^{2} / 2 t r^{2}$, ( $r$ being the distance between molecules). Using $t=0.123 \mathrm{eV}$ and $r \approx 3 \AA$ for BTQBT, we obtain $m^{*}=3 m$. By substituting this relation for Eq. (3), the mobility is evaluated as follows:

$$
\mu>6.7\left[\mathrm{~cm}^{2} \mathrm{~V}^{-1} \mathrm{~s}^{-1}\right] \text {. }
$$

This value is of the same order as the observed $3 \mathrm{~cm}^{2} \mathrm{~V}^{-1} \mathrm{~s}^{-1}$ for BTQBT.

The Hall effect measurement gives additional information about the sign of carriers. Its sign is found to be positive, which indicates that electrical conduction is dominated by hole carriers. The positive sign is consistent with the sign of $+500 \mu \mathrm{V} \mathrm{K}^{-1}$ at room temperature in the thermoelectric power measurement.

Since the numbers of electron and hole carriers are equal in an intrinsic semiconductor, a Hall voltage is zero by cancellation if the hole mobility $\left(\mu_{\mathrm{h}}\right)$ is equal to the electron mobility $\left(\mu_{\mathrm{e}}\right)$. The observed positive Hall voltage in BTQBT seems to result from $\mu_{\mathrm{h}}>\mu_{\mathrm{e}}$. If the observed IIall mobility could be separated into $\mu_{\mathrm{h}}$ and $\mu_{\mathrm{e}}$, we would expect a higher mobility for BTQBT. 


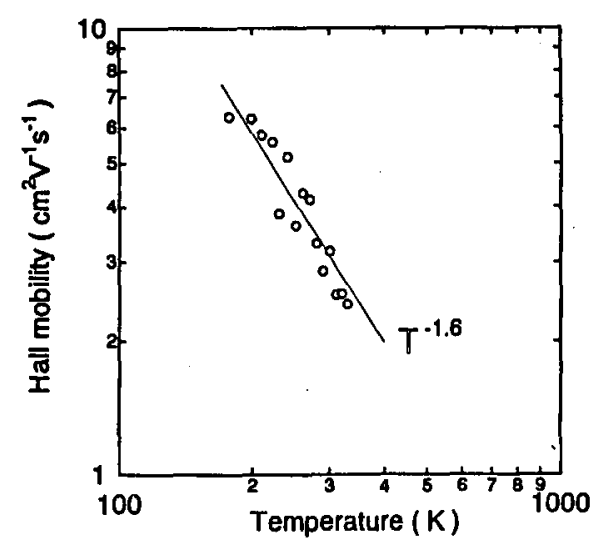

Fig. 4. Temperature dependence of the Hall mobility of BTQBT crystal. The solid line is drawn by the least-squares method.

We have examined the temperature-dependent IIall mobility of BTQBT [5]. The IIall voltages could be measured reliably in the temperature range 175 to $330 \mathrm{~K}$. Figure 4 shows the logarithmic plot of $\mu_{\mathrm{H}}$ vs. T. $\mu_{\mathrm{II}}$ increases linearly from $2.4 \mathrm{~cm}^{2} \mathrm{~V}^{-1} \mathrm{~s}^{-1}$ at $330 \mathrm{~K}$ to $6.3 \mathrm{~cm}^{2} \mathrm{~V}^{-1} \mathrm{~s}^{-1}$ at $175 \mathrm{~K}$ with decreasing temperature. The least-squares fitting gives an exponent $n$ of about -1.6 for the formula of $\mu_{\mathrm{H}} \propto T^{n}$.

We now discuss on the physical meaning of the $T^{-1.6}$ dependence. In the 1950 's the mobility of inorganic semiconductors such as silicon and germanium has been investigated extensively from both aspects of experiment and theory. The mobility is determined by the scattering process of conduction carriers. Three types of scattering have been studied theoretically: lattice [11], ionized and neutral impurities $[12,13]$, and dislocation [14]. Taking account of the additivity of the reciprocal relaxation time for each scattering, the temperature dependence of the reciprocal mobility is given by

$$
1 / \mu=\alpha_{l} T^{3 / 2}+\alpha_{i} T^{-3 / 2}+\alpha_{d} T^{-1},
$$

where the $\alpha$ 's are the temperature-independent parameters and the subscripts $l, i, d$ denote the scatterings by the lattice, impurity and dislocation. The contribution from the scatterings by ionized and neutral impurities would be negligible in the BTQBT crystal since the crystals consist of a single compound. Even if some impurities such as oxygen and nitrobenzene molecules are included in the crystal, the latter two types of scatterings are predominant only at low temperatures. In the high-temperature region, the lattice scattering mainly determines the mobility. Bardeen and Shokley derived a formula for the lattice-scattering mobility, $\mu_{\mathrm{L}}$,

$$
\mu_{\mathrm{L}}=\left(\frac{\sqrt{8 \pi}}{3} \frac{\hbar^{4} c_{l}}{E_{1}\left(m^{*}\right)^{5 / 2} k_{\mathrm{B}}^{3 / 2}}\right) T^{-3 / 2}
$$

where $c_{l}$ is the elastic constant and $E_{1}$ the deformation potential constant [12]. The observed $T^{-1.6}$ dependence corresponds to the theoretical $T^{-1.5}$ dependence, so 
that the temperature-dependent IIall mobility for the BTQBT crystal is explained in terms of lattice scattering.

In contrast to copper phthalocyanine reported by IIeilmeier and Harrison [15], for BTQBT the positive sign of carriers and the activation energy in the resistivity are not changed over the whole temperature range. These results imply that $\mu_{\mathrm{h}}$ is essentially larger than $\mu_{\mathrm{e}}$. It is concluded that the observed value of $3 \mathrm{~cm}^{2} \mathrm{~V}^{-1} \mathrm{~s}-1$ for the BTQBT crystal is not underestimated by partial cancellation.

\section{Energy band dispersion treatment}

The energy-band dispersion relation, $E=E(k)$, is a fundamental basis for understanding the basic properties of solids, such as electrical and optical properties. The typical method to observe the valence band dispersion directly is angle-resolved ultraviolet photoelectron spectroscopy (ARUPS) using synchrotron radiation. This method has been established for inorganic solids. However, for ordinary organic solids, the energy-band dispersion was difficult to observe. A few experimental works have been reported: Seki and his co-workers and also Ueno et al. reported the observation of intermolecular energy-band dispersion of $\sigma$-bands for oriented thin films of long chain organic compounds, hexatriacontane $\mathrm{CH}_{3}\left(\mathrm{CII}_{2}\right)_{34} \mathrm{CH}_{3}[16,17]$ and cadmium arachidate $\left[\mathrm{CII}_{3}\left(\mathrm{CH}_{2}\right)_{18} \mathrm{COO}\right]_{2} \mathrm{Cd}[18]$. II.C. Wolf's school reported the intermolecular energy-band dispersion of $\pi$-bands for dicyanoquinonediimine (DCNQI)-metal complex [19, 20].

\subsection{Photon energy dependence of normal emission spectra of BTQBT}

Recently, we have succeeded to observe directly the intermolecular $\pi$-band dispersion in the BTQBT well oriented thin films. The ARUPS measurements were carried out at an ultraviolet synchrotron orbital radiation facility in the Institute for Molecular Science. Figure 5 shows an example of the $h \nu$ dependence of photoelectron spectra for the oriented thin films (thickness $\approx 30 \AA$ ) of BTQBT. The two bands $A$ and $B$ are well separated from other valence bands, and assigned to be $b_{1 u}$ (IIOMO) and $b_{3 g}$ (NIIOMO) $\pi$ molecular orbitals in $D_{2 h}$ symmetry, respectively. For HOMO band, the continuous change of peak position with incident light $h \nu$ is clearly seen. At $h \nu=12 \mathrm{eV}$, the binding energy of the IIOMO peak is about $5.4 \mathrm{eV}$ from the vacuum level. With increasing photon energy, the peak position shifts to the higher binding energy side, and it turns back at $20 \mathrm{eV}$. At $48 \mathrm{eV}$, the peak exhibits the lowest binding energy, and shifts again to the higher binding energy side with further increasing photon energy. The total energy shift in the peak position is about $0.5 \mathrm{eV}$. The two extrema of peak positions at $h \nu=20 \mathrm{eV}$ and $48 \mathrm{eV}$ correspond to the edges of the Brillouin zone.

\subsection{Intermolecular energy-band dispersion}

Figure 6 shows the intermolecular energy-band dispersion for oriented thin films of BTQBT in the reduced zone scheme. The filled circles are the experimental 


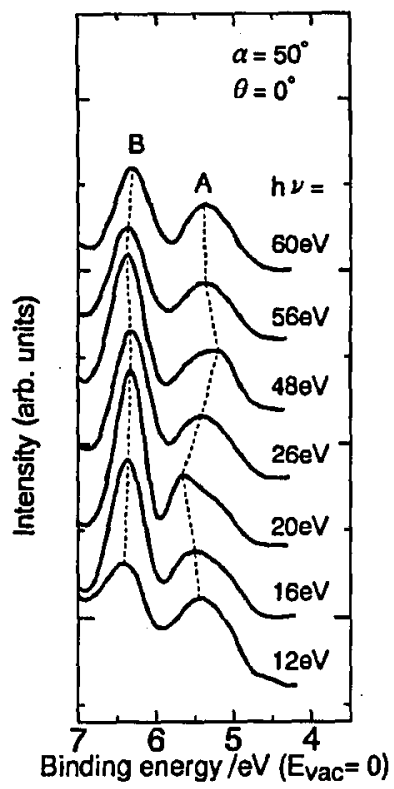

Fig. 5. Photon energy dependence of photoelectron spectra at normal emission for BTQBT thin films. The binding energy region is $4 \div 7 \mathrm{eV}$. The HOMO and NHOMO bands are labelled $A$ and $B$.

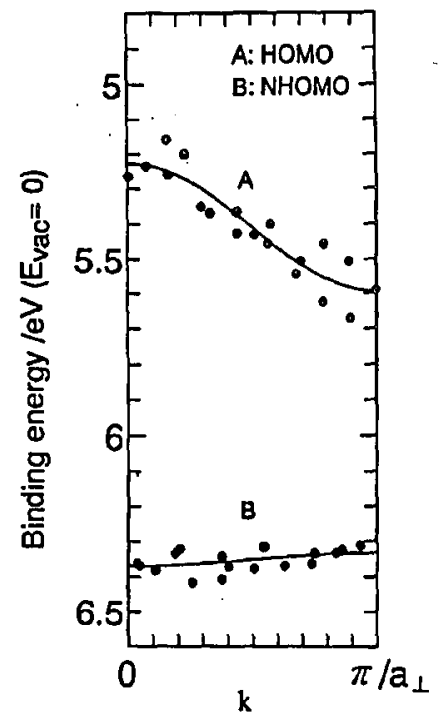

Fig. 6. Intermolecular energy-band dispersion for BTQBT thin films in the reduced zone scheme, where $a_{\perp}$ is $3.4 \AA$. The HOMO and NIOMO bands are indicated by $A$ and $B$. The solid line represents the best fit curve. 
results and in the solid line at the IIOMO band represents the best fit dispersion curve, where the transfer integral $\iota_{\text {HOMO }}$ is $0.092 \mathrm{eV}$. According to the energy-band calculation based on the extended IIückel method and tight-binding approximation, the transfer integral along the molecular stacking direction is $0.123 \mathrm{eV}$ for the IIOMO. It is widely accepted that a binding energy calculated by the extended Ilückel method tends to be much larger than an experimental result, and the calculated result should be compared with the experimental one in a contracted-and-shifted binding energy scale. Taking this into account, these calculated valucs correspond well to the experimental ones. Since the bandwidth $W$ is given by $4 l$ in the tight-binding model, the experimental bandwidth of the HOMO band becomes $\approx 0.4 \mathrm{eV}$, which is extraordinarily large as compared to those in usual organic solids.

These results demonstrated that the single component organic semiconductor, BTQBT, has a strong intermolecular interaction, which can be derived from the introduction of a covalent interaction between chalcogen atoms in addition to the usual interaction by van der Waals forces.

\section{Acknowledgments}

These works have been done with Drs. S. IIasegawa, T. Mori, S. Tanaka, Y. Yamashita, I. Fujimoto, K. Seki and N. Ueno. The authors acknowledge them.

\section{References}

[1] Y. Yamashita, Y. Kobayashi, T. Miyasli, Angew. Chem. Int. Ed. Engl. 28, 1052 (1989).

[2] R. Neidlein, D. Tran-Viet, A. Gieren, M. Kokkinidis, R. Wilckens, H. Geserich, W. Ruppel, Chem. Ber. 115, 2898 (1982).

[3] Y. Yamashita, S. Tanaka, K. Imaeda, H. Inokuchi, Chem. Lett., 1213 (1991).

[4] K. Imaeda, Y. Yamashita, Y. Li, T. Mori, H. Inokuchi, M. Sano, J. Mater. Chem. 2, 115 (1992).

[5] K. Imaeda, Y. Li, Y. Yamashita, H. Inokuchi, M. Sano, J. Mater. Chem. 5, 861 (1995).

[6] L.B. Schein, D.W. Brown, Mol. Cryst. Liq. Cryst. 87, 1 (1982).

[7] Y. Maruyama, Mol. Cryst. Liq. Cryst. 171, 287 (1989).

[8] T. Mori, private communication.

[9] S. Hasegawa, T. Mori, K. Imaeda, S. Tanaka, Y. Yamashita, H. Inokuchi, H. Fujimoto, K. Seki, N. Ueno, J. Chem. Phys. 100, 6969 (1994).

[10] H. Meier, Organic Semiconductors, Verlag Chemie, Weinheim 1974.

[11] J. Bardeen, W. Shockley, Phys. Rev. 80, 72 (1950).

[12] E. Conwell, V.F. Weisskopf, Phys. Rev. 77, 388 (1950).

[13] C. Erginsoy, Phys. Rev. 79, 1013 (1950).

[14] D.L. Dexter, F. Seitz, Phys. Rev. 86, 964 (1952).

[15] G.H. Heilmeier, S.E. Harrison, Phys. Rev. 132, 2010 (1963).

[16] K. Seki, U.O. Karlsson, R. Engelhaldt, E.E. Koch, Chem. Phys. Lelt. 103, 343 (1984). 
[17] K. Seki, N. Ueno, U.O. Karlsson, R. Engelhaldt, E.E. Koch, Chem. Phys. 105, 247 (1986).

[18] N. Ueno, W. Gaedeke, E.E. Koch, R. Engelhaldt, R. Dudde, L. Laxhuber, H. Moehwald, J. Mol. Electron. 1, 19 (1985).

[19] D. Schmeisser, W. Jaegermann, C. Pettenkofer, II. Wachtel, A. Jimenez-Gonzales, J.U. Schütz, H.C. Wolf, P. Erk, H. Meixner, S. Hünig, Solid State Commun. 81, 827 (1992).

[20] H. Wachtel, J.U. Schütz, H.C. Wolf, Synlh. Mel. 41-43, 1789 (1991). 